\title{
The impact of pretransplant hepatic encephalopathy, model for end-stage liver disease (MELD) scale on long-term survival following deceased donor liver transplantation: a retrospective study
}

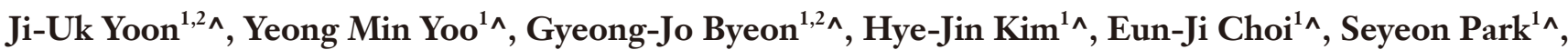 \\ Hee Young $\mathrm{Kim}^{1} \wedge$
}

${ }^{1}$ Department of Anesthesia and Pain Medicine, Pusan National University Yangsan Hospital, Yangsan, Republic of Korea; ${ }^{2}$ Department of Anesthesia and Pain Medicine, School of Medicine, Pusan National University, Yangsan, Republic of Korea

Contributions: (I) Conception and design: JU Yoon, HY Kim; (II) Administrative support: HY Kim; (III) Provision of study materials or patients: YM Yoo, HY Kim; (IV) Collection and assembly of data: YM Yoo, HY Kim; (V) Data analysis and interpretation: YM Yoo, HY Kim; (VI) Manuscript writing: All authors; (VII) Final approval of manuscript: All authors.

Correspondence to: Hee Young Kim. Department of Anesthesia and Pain Medicine, Pusan National University Yangsan Hospital, 20, Geumo-ro, Beomeo-ri, Mulgeumeup, 50612, Yangsan, Republic of Korea. Email: anekhy@gmail.com.

\begin{abstract}
Background Liver transplantation (LT) has the incidence of 30-day mortality about 5-10\%, Jo et al. reported that 30-day mortality and 1-year mortality for DDLT were 30\%, and 39\% respectively. It is not easy to create a model for predicting post-transplantation outcomes based on pretransplant variables. MELD does not take into account individual complications such as hepatic encephalopathy (HE), and research has shown that the greater the severity of pretransplant HE, the lower the survival rate after LT; the importance of monitoring for $\mathrm{HE}$ is therefore emphasized.

Methods: The medical records of adult patients who underwent deceased donor LT (DDLT) were retrospectively reviewed for analysis of the effect of $\mathrm{HE}$ on the long-term survival rate of post-transplant for more than 1 year.

Results: Presence of HE is not statistically associated to patient survival $(\mathrm{P}=0.062)$, but the hazard ratio is 1.954 (95\% CI, 0.968, 3.943). In addition, the severe HE group significantly decreased survival compared to the non-HE group, and the cumulative 1- and 3-year overall survival rates were $80.9 \%$ and $78.7 \%$, respectively, in non $\mathrm{HE}$ group, and $65.7 \%$ and $56.1 \%$, respectively, in severe $\mathrm{HE}$ group $(\mathrm{P}=0.031)$.

Conclusions: Severe HE is a factor influencing the long-term survival over 3 years in the patients who underwent DDLT. Although prospective validation should be conducted to determine the prognostic value of $\mathrm{HE}$ severity, efforts could be made to reduce the severity of HE before DDLT, and consider severity of HE rather than MELD score in DDLT allocation.
\end{abstract}

Keywords: End stage liver disease; hepatic encephalopathy (HE); liver transplantation (LT); mortality; survival

Submitted Jan 04, 2021. Accepted for publication Mar 31, 2021.

doi: 10.21037/apm-21-21

View this article at: http://dx.doi.org/10.21037/apm-21-21

^ ORCID: Ji-Uk Yoon, 0000-0002-3971-2502; Yeong Min Yoo, 0000-0003-3536-0447; Gyeong-Jo Byeon, 0000-0001-5333-3894; Hye-Jin Kim, 0000-0003-1630-0422; Eun-Ji Choi, 0000-0003-3731-0785; Seyeon Park, 0000-0001-7183-1811; Hee Young Kim, 0000-0001-78098739. 


\section{Introduction}

Hepatic encephalopathy (HE) is a serious complication of non-compensated end-stage liver disease and exhibits a wide range of neuropsychiatric abnormalities ranging from subtle psychological alterations to coma. HE is reversible in patients with no neurological or metabolic abnormalities (1), but liver transplantation (LT) is a definitive treatment for HE (2).

LT has a 30 -day mortality of about $5-10 \%$ (3), and Jo et al. reported that 30-day mortality and 1-year mortality for DDLT were $30 \%$, and $39 \%$ respectively in their study (3). It is very important to identify pretransplant risk factors for early mortality, but it is difficult to create a model for predicting post-transplant outcomes based on pretransplant variables. There are also variables independent of pretransplant factors, such as level of surgical skill, adverse events in the perioperative period, graft rejection, and biliary and vascular complications. Nevertheless, there are studies that predict post-transplant survival using the model for end-stage liver disease (MELD) score (4-6). Habib et al. reported that recipient age $>65$ years, retransplantation, MELD score $>25$, non-cholestatic etiology of primary disease, and Child-Pugh (C-P) C status were independent predictors for patient survival at 1 year, but the influence of MELD score on 1-year patient survival is lost beyond the first year (4).

In addition, MELD does not take into account individual complications such as hepatic portal hypertension, hepatorenal syndrome, hepatopulmonary syndrome (HPS), and HE (7). Research has shown that the greater the severity of pretransplant HE, the lower the survival rate, and the higher the infection rate after LT; the importance of monitoring for $\mathrm{HE}$ is therefore emphasized $(3,8)$.

The purpose of this study was to analyse the association of $\mathrm{HE}$ with a long-term post-transplant survival rate of more than 1 year in patients who underwent deceased donor LT (DDLT), and to determine whether pretransplant HE can be used as a predictor of long-term survival. We present the following article in accordance with the STROBE reporting checklist (available at http://dx.doi.org/10.21037/ apm-21-21).

\section{Methods}

\section{Study population}

The medical records of adults (age $>18$ years) who underwent DDLT at Pusan National University Yangsan
Hospital, Yangsan, Korea, from January 1, 2010, to December 31, 2015, were retrospectively reviewed. We received approval of waived consent from the Institutional Review Board of Pusan National University Yangsan Hospital due to the retrospective nature of the study (Ref: 05-2018-183). The study was conducted in accordance with the Declaration of Helsinki (as revised in 2013).

\section{Evaluation of MELD score and severity of $\mathrm{HE}$}

The pretransplant MELD score and the severity of HE were measured based on the patient's documented level of consciousness and the results of blood tests one day before transplant. The MELD score was calculated using the United Network of Organ Sharing formula, which includes serum total bilirubin level, serum creatinine, and international normalized ratio (INR) (available at http:// www.unos.org/resources):

MELD score $=9.57 \times \log _{\mathrm{e}}$ creatinine $(\mathrm{mg} / \mathrm{dL})+3.78 \times$ $\log _{\mathrm{e}}$ bilirubin $(\mathrm{mg} / \mathrm{dL})+11.20 \times \log _{\mathrm{e}} \mathrm{INR}+6.43$.

$\mathrm{HE}$ assessed by the hepatologist immediately before surgery was reviewed, and if there was no description of HE, its pretransplant severity was classified by the data reviewer using the "gold standard" West-Haven criteria [grade 0, no HE; grade 1, euphoria or anxiety, shortened attention span, trivial lack of awareness, impaired performance of addition or subtraction; grade 2, lethargy or apathy, minimal disorientation for time or place, subtle personality change, inappropriate behaviour; grade 3, somnolence to semi stupor (but responsive to verbal stimuli), confusion, gross disorientation; grade 4, coma (unresponsive to verbal or noxious stimuli)] (9). Medical history, blood tests, and imaging studies were reviewed to help rule out changes in consciousness or cognition resulting from medications, alcohol abuse, drug use, hyponatremia, and psychiatric disease. The primary end point of this study was to find the effect of severity of $\mathrm{HE}$ on post-transplant survival. The HE group was further classified into mild HE (grade I-II) and severe HE (grade III-IV) groups. Our secondary end point was prediction of long-term survival based on HE severity.

\section{Statistical analysis}

The independent $t$-test or Wilcoxon rank sum test was used for statistical analysis of continuous data, and Fisher's exact test was used to assess categorical data. For multivariable analyses, we used the Cox proportional hazards regression 
method to determine the risk factors for low survival rates. Survival curves were generated using the KaplanMeier method, and the log-rank test was used to compare survival. Spearman's correlation analysis was used to assess correlation between grade of HE and MELD score. We assessed the ability of HE grade to predict mortality in the DDLT patients by measuring the concordance (c-statistic) equivalent to the area under the receiver operating characteristic curve (ROC). A c-statistic between 0.8 and 0.9 indicates excellent diagnostic accuracy, and a model with a c-statistic over 0.7 should be considered clinically useful $(10,11)$. The variables with $\mathrm{P}$ values $<0.05$ were considered statistically significant. $\mathrm{P}<0.05$ is two-sided. All statistical analyses were performed using $\mathrm{R}$ 3.5.0 software (www. r-project.org).

\section{Results}

\section{Characteristics of patients and mortality}

A total of 114 adult patients underwent DDLT during the study period. Four were excluded due to incomplete data; therefore, the study was conducted with a total of 110 patients. The mean patient age was 55 (range, 48.25-62.00) years, and the proportion of male was higher than that of female. The average MELD score was 30.5 (range, 23.25$35.00)$, with no difference between the HE group ( $n=63$ ) and the non-HE $(n=47)$ group. The aetiology of liver failure was most frequently viral hepatitis $(52,47.3 \%)$, followed by alcohol use, autoimmune disease, and cholestasis. The demographic features of the patients in the $\mathrm{HE}$ and nonHE groups were not statistically different, but the C-P score, INR, serum sodium level, duration of mechanical ventilation, and mortality were significantly higher in the HE group compared to the non-HE group. The mortality of the HE group $(27,42.9 \%)$ was also significantly higher than that of the non-HE group $(11,23.4 \%)(\mathrm{P}=0.043)$, but the causes of death were not statistically different (Table 1). A total of 38 patients died after DDLT, and the causes of death were infection $(8,21.1 \%)$, acute rejection $(5,13.2 \%)$, pulmonary complications $(5,13.2 \%)$, cerebrovascular accident $(4,10.5 \%)$, and chronic rejection $(2,5.3 \%)$. The HE group was subdivided into the mild HE group (grade I-II) and the severe HE group (grade III-IV); there were $22(34.9 \%)$ and $41(65.1 \%)$, respectively. To examine the correlation between the grade of HE and MELD score, Spearman's correlation analysis was performed, but the correlation coefficient $(0.13)$ was not statistically significant
(Figure 1).

\section{Univariate survival analysis}

Mean duration of follow-up was 4.07 (range, 0.52-5.60) years, and overall survival was $65.5 \%$. In univariate analyses (Table 2), we found that age over 65 years, grade of HE, severe HE, presence of HPS, and duration of mechanical ventilation were significantly associated with patient survival. Hazard ratios (HRs) for advanced age, severe HE, and presence of HPS were 2.291 (95\% CI, 1.0834.845), 2.245 (95\% CI, 1.074-4.690), and 4.833 (95\% CI, 1.695-13.782), respectively. The presence of $\mathrm{HE}$ was not statistically associated with patient survival $(\mathrm{P}=0.062)$, but the HR was 1.954 (95\% CI, 0.968-3.943), indicating about twice the risk of death compared to the non-HE group. In addition, the severe HE group had significantly decreased survival compared to the non-HE group; the cumulative 1 - and 3 -year overall survival rates were $80.9 \%$ and $78.7 \%$, respectively, in non-HE group, and $65.7 \%$ and $56.1 \%$, respectively, in the severe HE group $(\mathrm{P}=0.031)$ (Figure 2). On the other hand, pretransplant MELD score and C-P score were not associated with survival.

\section{Multivariable survival analysis}

Using the Cox proportional hazard model and after adjustment for age and gender (Table 3), we found that severe HE was significantly associated with poor patient survival (HR 2.137; 95\% CI, 1.018-4.485). However, the area under the receiver operating curve (ROC) for the grade of $\mathrm{HE}$ and mortality was 0.63 (Figure 3).

\section{Discussion}

Our study demonstrates that severe HE is associated with patient survival after DDLT and HR of death is about twice compared to the non-HE group although HE is not able to be used for the predictor of post-transplant mortality.

MELD-based systems for prioritization of LT allocation were introduced in 2002 (4). However, HE is not being used as a criterion for prioritization of patients with chronic liver disease awaiting LT. For validation of the scoring system for allocation of LT, there have been many studies on the effect of MELD score on post-transplant mortality $(4,5,12)$.

However, it is difficult to predict a post-transplant outcome based on pretransplant variables because of other perioperative factors such as level of surgical skill, 
Table 1 Demographic, clinical, and biochemical features in patients who underwent DDLT

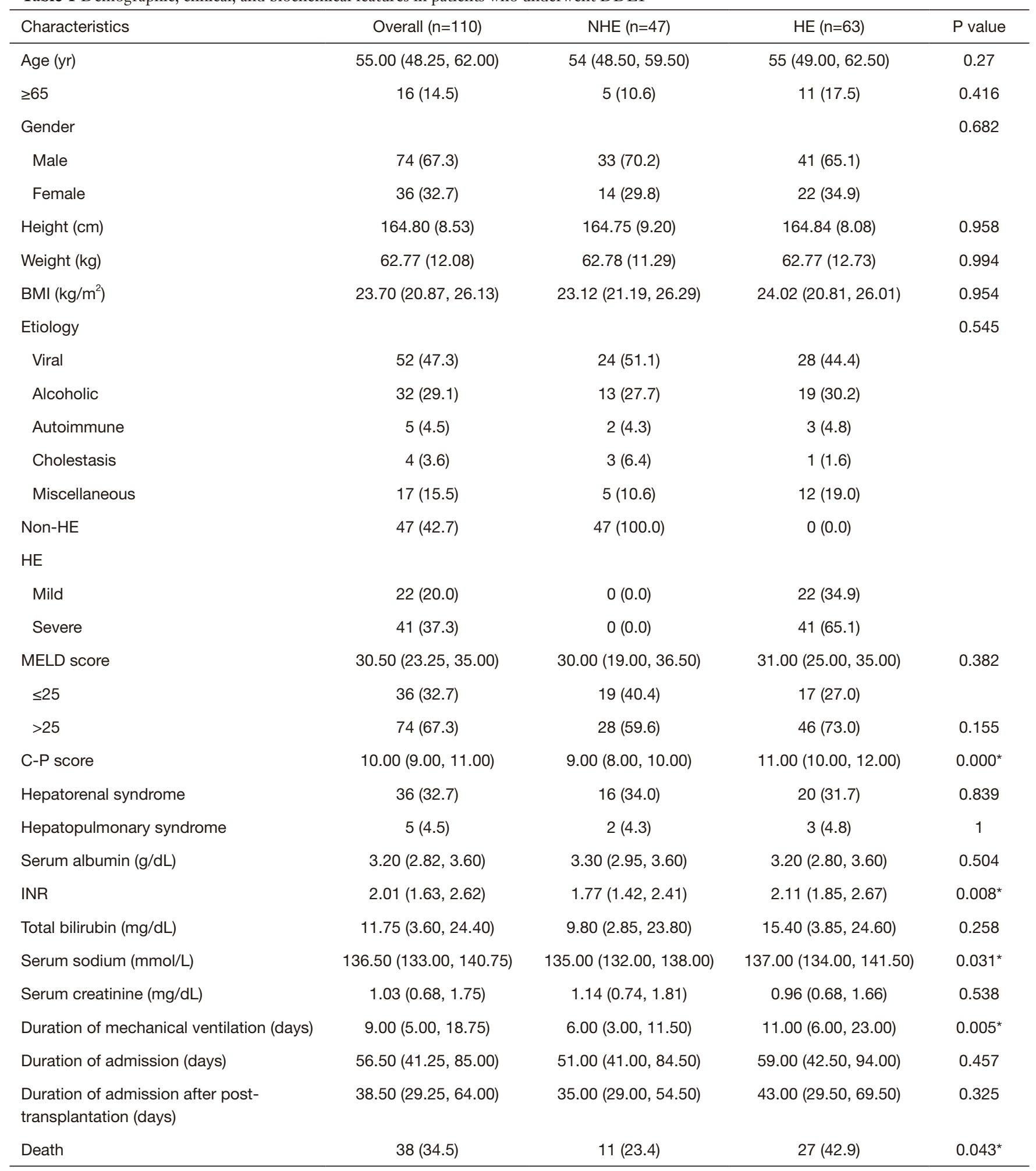

Table 1 (continued) 
Table 1 (continued)

\begin{tabular}{lccc}
\hline Characteristics & Overall $(\mathrm{n}=110)$ & NHE $(\mathrm{n}=47)$ & $\mathrm{HE}(\mathrm{n}=63)$ \\
\hline Cause of death & & & 0.584 \\
Acute rejection & $5(13.2)$ & $0(0.0)$ & $5(18.5)$ \\
Chronic rejection & $2(5.3)$ & $0(0.0)$ & $2(7.4)$ \\
Infection & $8(21.1)$ & $3(27.3)$ & $5(18.5)$ \\
Pulmonary complication & $5(13.2)$ & $1(9.1)$ & $4(14.8)$ \\
Cerebrovascular accident & $4(10.5)$ & $1(9.1)$ & $3(11.1)$ \\
Others & $14(36.8)$ & $6(54.5)$ & $8(29.6)$ \\
\hline
\end{tabular}

Categorical variables expressed as number (\%), non-normal continuous variables as median (Q1-Q3) and normal continuous variables as mean $\pm \mathrm{SD}$. *, P<0.05. BMI, body mass index; C-P, Child-Pugh; DDLT, deceased-donor liver transplantation; HE, hepatic encephalopathy; INR, international normalized ratio; MELD, model for end-stage liver disease; NHE, non-hepatic encephalopathy.

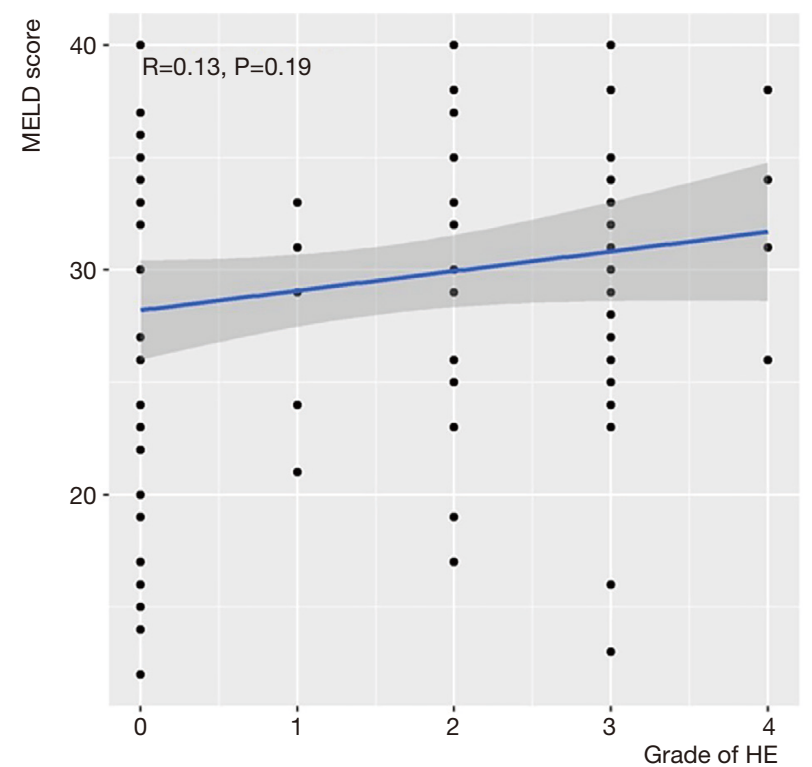

Figure 1 Correlation between grade of hepatic encephalopathy and MELD score in the patients who underwent DDLT. Spearman's correlation analysis was carried out. DDLT, deceased donor liver transplantation; HE, hepatic encephalopathy; MELD, model for end-stage liver disease.

graft rejection, and biliary and vascular complications. Habib et al. reported that a high pretransplant MELD score ( $\geq 26)$ was associated with poor outcome in patients who underwent DDLT, but the effect disappeared 1 year after transplantation (4). Saab et al. (5) and Onaca et al. (12) similarly reported that a higher MELD score was associated with lower 1-year survival, but they did not report an association with long-term survival or using MELD as a survival predictor. Although the MELD score is a convenient, reproducible, and objective tool for LT allocation using biochemical variables such as serum bilirubin, serum creatinine, and INR, it has limitations because it was designed to predict outcome after the transjugular intrahepatic portosystemic shunt procedure in chronic liver disease (13). First, complications of portal hypertension such as oesophageal variceal bleeding, spontaneous bacterial peritonitis, ascites, and portosystemic encephalopathy are not taken into account. Second, organ allocation favours sicker patients when using the MELD score, and this can lead to early post-transplant deaths and wasting of donor livers (7). Third, although the MELD score is objective, creatinine (one of the variables used) is affected by factors such as co-morbid illnesses, muscle mass, age, and exposure to nephrotoxic drugs, contrast agents, or diuretics (14).

$\mathrm{HE}$ is a complication of portosystemic shunts associated with liver failure and cirrhosis (15). Its neurochemical and neurophysiological mechanisms are unknown, but ammonia is considered an important factor (16). HE is characterized by cognitive impairment and deficits of motor accuracy and speed (17), and it occurs in $35-45 \%$ of patients undergoing orthotopic LT (OLT) (18). Neurologic complications such as encephalopathy, seizures, tremor, psychosis, and posterior reversible encephalopathy syndrome may occur in $30 \%$ of patients undergoing OLT, leading to high morbidity and prolonged hospital stays $(19,20)$. Although some studies have shown that $\mathrm{HE}$ is not an independent factor affecting short-term survival in patients with cirrhosis (21), studies 
Table 2 Factors associated with overall survival of patients who underwent DDLT (univariate analysis)

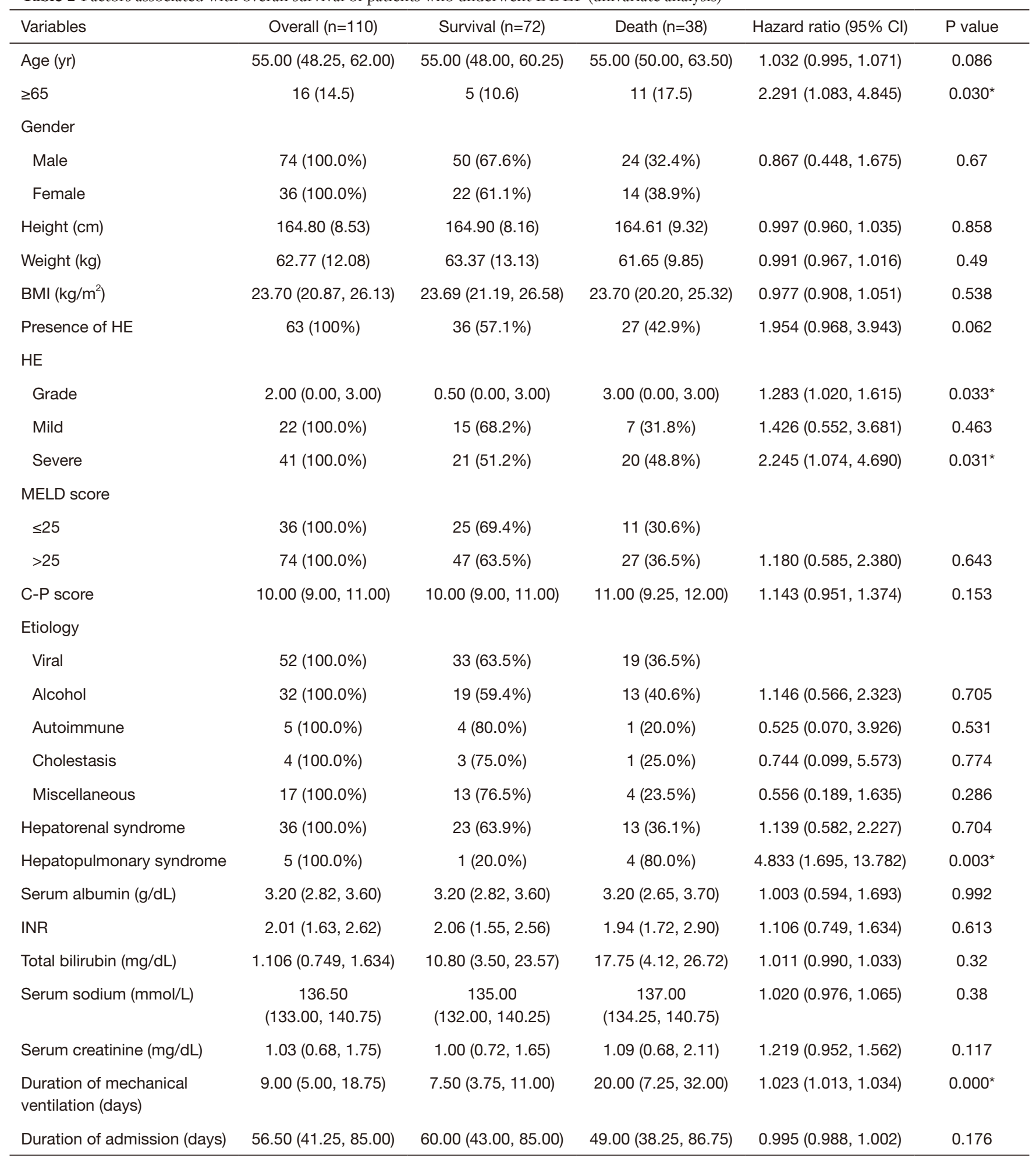

Categorical variables expressed as number (\%), non-normal continuous variables as median (Q1-Q3) and normal continuous variables as mean \pm SD. * $\mathrm{P}<0.05$. BMI, body mass index; C-P, Child-Pugh; Cl, confidence interval; DDLT, deceased-donor liver transplantation; HE, hepatic encephalopathy; INR, international normalized ratio; MELD, model for end-stage liver disease; NHE, non-hepatic encephalopathy. 


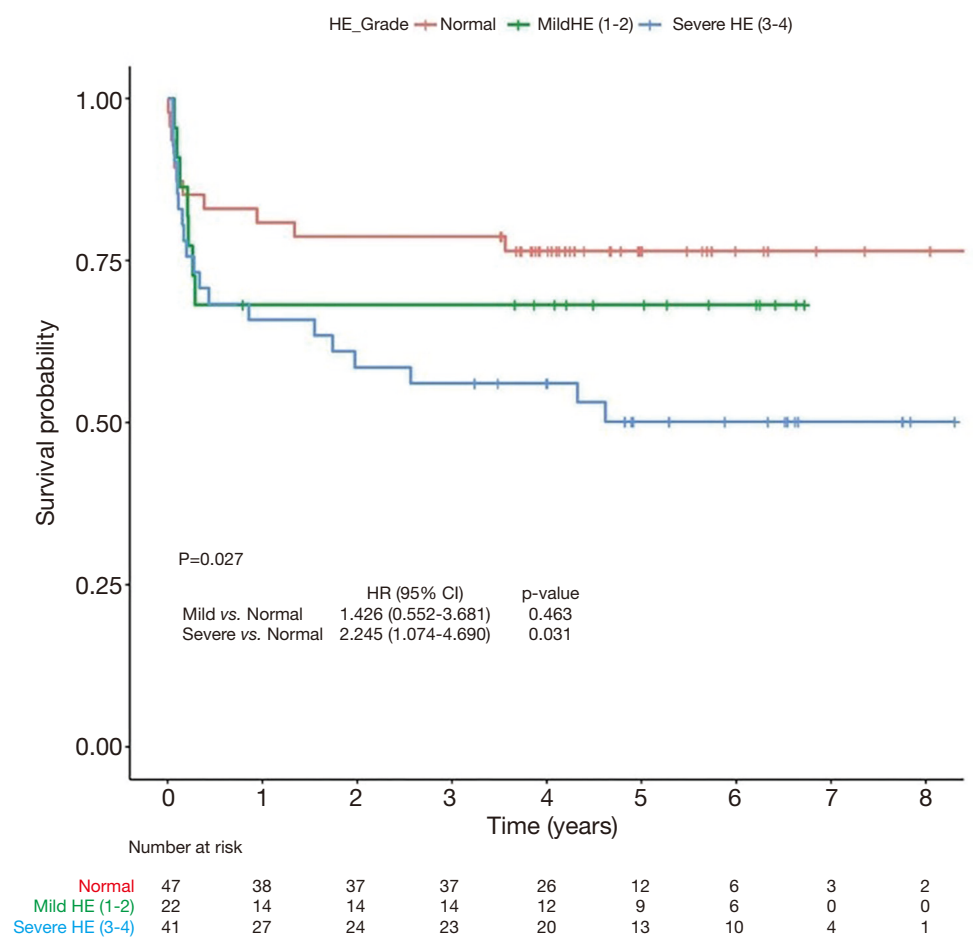

Figure 2 Survival probability based on severity of hepatic encephalopathy in the patients who underwent DDLT. The red line indicates the non HE group, the green line indicates the mild HE (grade I, II) group, and the blue line indicates the severe HE (grade III, IV) group. Kaplan-Meier analysis with comparison among groups by log-rank test was carried out. The cumulative 1- and 3-year overall survival rates were $80.9 \%$ and $78.7 \%$, respectively, in non HE group, and $65.7 \%$ and 56.1\%, respectively, in severe HE group (P=0.031). DDLT, deceased donor liver transplantation; HE, hepatic encephalopathy.

Table 3 Hazard ratio of hepatic encephalopathy on overall mortality of patients who underwent DDLT (multivariable analysis), with adjustment for the gender and age

\begin{tabular}{lll}
\hline Variables & Hazard ratio $(95 \% \mathrm{Cl})$ & P value \\
\hline $\mathrm{HE}$ & & \\
Mild & $1.312(0.506,3.402)$ & 0.576 \\
Severe & $2.137(1.018,4.485)$ & $0.045^{*}$ \\
\hline
\end{tabular}

*, $\mathrm{P}<0.05$. Cl, confidence interval; DDLT, deceased-donor liver transplantation; $\mathrm{HE}$, hepatic encephalopathy.

have proven that $\mathrm{HE}$ is useful as a predictor of survival in end-stage liver disease $(22,23)$. Among these, studies were conducted on the effect of $\mathrm{HE}$ on post-transplant morbidity and mortality in order to overcome the limitations of the MELD score. According to You et al., the sequelae of HE are associated with severe HE (grade III, IV), and aggressive treatment of HE prior to LT may improve outcomes (2). In addition, Jo et al. reported that pretransplant HE is significantly related to 30 -day mortality in DDLT, and that $\mathrm{HE}$ can be used as a predictor of survival (3).

According to our study, the mortality of the HE group was significantly higher than that of the non-HE group, and the hazard ratio for mortality with the presence of $\mathrm{HE}$ was about twice that of the non-HE group. In addition, survival was significantly decreased in the severe HE group compared to the non-HE group. It was not possible to prove $\mathrm{HE}$ as a predictor for mortality in DDLT, because the area under the ROC for the grade of HE and mortality was 0.63 .However, the HR for mortality, which is more than twice as high in the severe HE group as in the nonHE group, suggests clinical significance of HE severity in DDLT. Wong et al. suggested that grade III-IV HE at the time of LT is related to lower survival. However, via status 1A prioritization, they excluded patients undergoing LT for fulminant liver failure, and their study setting was different from ours (24). Stewart et al. reported that using overt HE can improve MELD's prognostic accuracy (22), and this supports the results of our study. As in the results of the 


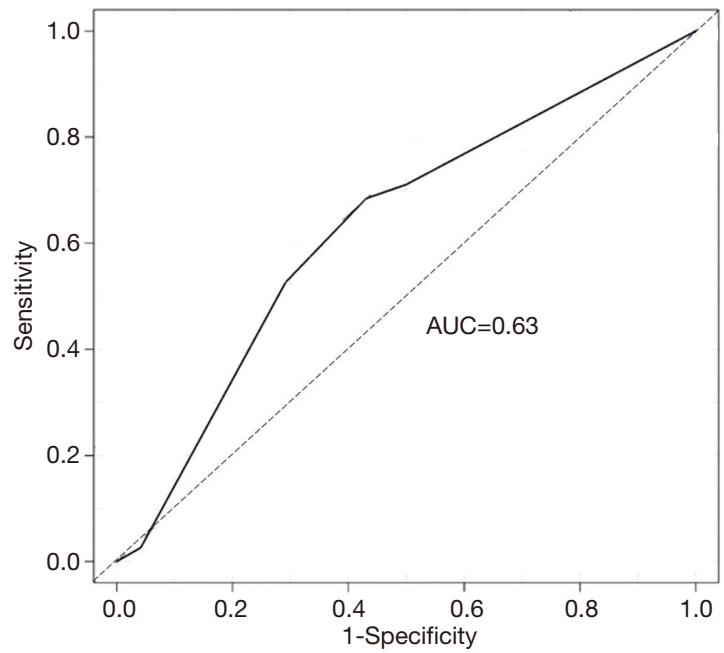

Figure 3 The area under the receiving operating curve (ROC) for the grade of $\mathrm{HE}$ with mortality. Dotted line represents the ROC based on chance alone and has a c-statistic of 0.5 . AUC, area under the curve; HE, hepatic encephalopathy.

study by You et al. (2), we showed no correlation between the MELD score and severity of HE in patients undergoing DDLT, and a MELD score $>25$ points had a mortality HR of 1.18, making it difficult to assume clinical significance. Therefore, in patients undergoing DDLT, regardless of the MELD score, severity of HE may further affect the patient's postoperative outcome. According to Garcia-Martinez et al., HE can cause permanent cognitive dysfunction after LT, and they also reported that permanent brain injury by $\mathrm{HE}$ can hinder recovery of consciousness and function of the respiratory centre, increasing the time of ventilation even though metabolic encephalopathy is corrected through LT (25). In our study, duration of mechanical ventilation in the HE group was 8 days longer than in the non-HE group. In addition, HE may improve with medical treatment in the early stages, but medical treatment becomes less effective if the severity of $\mathrm{HE}$ increases and non-compensated liver failure progresses. Some reports indicated that cognitive function recovers 3 months after LT $(26,27)$, but neurocognitive deficits such as memory impairment, psychomotor slowing, anxiety, and depression could remain (2). Therefore, pharmacologic therapy such as nonabsorbable disaccharides (e.g., lactulose) and non-systemic antibiotics (e.g. rifaximin) can be used in the gastrointestinal tract to reduce the systemic levels of toxins produced by ammonia, thereby reducing cerebral exposure, and this may improve post-transplant outcome (28).
However, there are problems in the assessment of HE severity. Some clinicians consider fatigue, occasional forgetfulness, and insomnia as HE symptoms, while others do not; in some cases, drowsiness caused by sedative medications is mistaken for a sign of HE (7). Therefore, it is necessary to objectively evaluate brain function using tools such as positron emission tomography and functional MR imaging.

Unlike previous studies, ours is a long-term survival study of more than 3 years in patients receiving DDLT in a single institution. The study results can be clinically applied as follows. First, measuring the objective brain function evaluation index using tools such as positron emission tomography and functional MR imaging during LT allocation should be considered. Second, in patients undergoing DDLT, the severity of HE can affect shortand long-term mortality, and there is a difference in the duration of postoperative ventilator use. It seems important to recognize the neurological changes of $\mathrm{HE}$ prior to transplant and to try to reduce the severity of $\mathrm{HE}$ through medical treatment.

There are several limitations to our retrospective study of HE. First, because HE is subject to the evaluator's judgment, different $\mathrm{HE}$ grades can be assigned to the same patient. In this study, some HE grades were assigned by the hepatologist immediately before surgery, but when there was no description of $\mathrm{HE}$ grade, the data reviewers assigned grade based on the clinical condition described in the medical record. Grade can be overestimated by the evaluator if severity of HE is applied to LT allocation, and a quantitative and objective grading system for HE must be established.

Second, treating HE may lessen its severity. Unfortunately, the effect of a change in HE severity on mortality was not investigated in this study. Nevertheless, because the severity of $\mathrm{HE}$ just before surgery affects posttransplant mortality, efforts to treat pretransplant $\mathrm{HE}$ appear to be important.

Third, we confirmed that severe HE affects mortality, but its usefulness as a predictor of post-transplant mortality could not be determined due to small sample size. Although there were no statistically significant differences, the mortality HR for HE was more than twice that of non$\mathrm{HE}$, and clinically significant. HE may prove useful as a predictor with a larger sample size. Therefore, additional data review is necessary.

In conclusion, our results demonstrated that severe HE influences survival over 3 years in patients who underwent 
DDLT. Clinically, efforts could be made to reduce the severity of HE before DDLT, and it may be necessary to consider severity of HE rather than MELD score in DDLT allocation. In addition, the sample size should be increased, and prospective validation should be conducted to determine the prognostic value of HE severity.

\section{Acknowledgments}

The authors thank the Department of Biostatics, Clinical Trial Center, Biomedical Research Institute, Pusan National University Hospital for their contributions to this work.

Funding: This study was supported by Research institute for Convergence of Biomedical Science and Technology (302017-011), Pusan National University Yangsan Hospital.

\section{Footnote}

Reporting Checklist: The authors have completed the STROBE reporting checklist. Available at http://dx.doi. org/10.21037/apm-21-21

Data Sharing Statement: Available at http://dx.doi. org/10.21037/apm-21-21

Conflicts of Interest: All authors have completed the ICMJE uniform disclosure form (available at http://dx.doi. org/10.21037/apm-21-21). HYK reports Research institute for Convergence of Biomedical Science and Technology (30-2017-011), Pusan National University Yangsan Hospital funding. The other authors have no conflicts of interest to declare.

Ethical Statement: The authors are accountable for all aspects of the work in ensuring that questions related to the accuracy or integrity of any part of the work are appropriately investigated and resolved. We received approval of waived consent from the Institutional Review Board of Pusan National University Yangsan Hospital due to the retrospective nature of the study (Ref: 05-2018183). The study was conducted in accordance with the Declaration of Helsinki (as revised in 2013).

Open Access Statement: This is an Open Access article distributed in accordance with the Creative Commons Attribution-NonCommercial-NoDerivs 4.0 International License (CC BY-NC-ND 4.0), which permits the noncommercial replication and distribution of the article with the strict proviso that no changes or edits are made and the original work is properly cited (including links to both the formal publication through the relevant DOI and the license). See: https://creativecommons.org/licenses/by-nc-nd/4.0/.

\section{References}

1. Ferenci P, Lockwood A, Mullen K, et al. Hepatic encephalopathy--definition, nomenclature, diagnosis, and quantification: final report of the working party at the 11th World Congresses of Gastroenterology, Vienna, 1998. Hepatology 2002;35:716-21.

2. You DD, Choi GS, Kim JM, et al. Long-term Outcomes for Liver Transplant Recipients in Terms of Hepatic Encephalopathy. Transplant Proc 2017;49:1425-9.

3. Jo YY, Choi YS, Joo DJ, et al. Pretransplant mortality predictors in living and deceased donor liver transplantation. J Chin Med Assoc 2014;77:16-20.

4. Habib S, Berk B, Chang CC, et al. MELD and prediction of post-liver transplantation survival. Liver Transpl 2006;12:440-7.

5. Saab S, Wang V, Ibrahim AB, et al. MELD score predicts 1-year patient survival post-orthotopic liver transplantation. Liver Transpl 2003;9:473-6.

6. Jacob M, Copley LP, Lewsey JD, et al. Pretransplant MELD score and post liver transplantation survival in the UK and Ireland. Liver Transpl 2004;10:903-7.

7. Wiesner R, Edwards E, Freeman R, et al. Model for endstage liver disease (MELD) and allocation of donor livers. Gastroenterology 2003;124:91-6.

8. Brandman D, Biggins SW, Hameed B, et al. Pretransplant severe hepatic encephalopathy, peritransplant sodium and post-liver transplantation morbidity and mortality. Liver Int 2012;32:158-64.

9. Ferenci P. Hepatic encephalopathy. Gastroenterol Rep (Oxf) 2017;5:138-47.

10. Wiesner RH, McDiarmid SV, Kamath PS, et al. MELD and PELD: application of survival models to liver allocation. Liver Transpl 2001;7:567-80.

11. Hanley JA MB. The meaning and use of the area under a receiver operating characteristic (ROC) curve. Radiology 1982;143:29-36.

12. Onaca NN, Levy MF, Sanchez EQ, et al. A correlation between the pretransplantation MELD score and mortality in the first two years after liver transplantation. Liver Transpl 2003;9:117-23.

13. Malinchoc M, Kamath PS, Gordon FD, et al. A model to predict poor survival in patients undergoing transjugular 
intrahepatic portosystemic shunts. Hepatology 2000;31:864-71.

14. Yoo HY ED, Thuluvath PJ. Relationship of the model for end-stage liver disease (MELD) scale to hepatic encephalopathy, as defined by electroencephalography and neuropsychometric testing, and ascites. Am J Gastroenterol 2003;98:1395-9.

15. Vilstrup H, Amodio P, Bajaj J, et al. Hepatic encephalopathy in chronic liver disease: 2014 Practice Guideline by the American Association for the Study of Liver Diseases and the European Association for the Study of the Liver. Hepatology 2014;60:715-35.

16. Grover VP, Tognarelli JM, Massie N, et al. The why and wherefore of hepatic encephalopathy. Int J Gen Med 2015;8:381-90.

17. Weissenborn K, Ennen JC, Schomerus H, et al. Neuropsychological characterization of hepatic encephalopathy. J Hepatol 2001;34:768-73.

18. Campagna F, Montagnese S, Schiff S, et al. Cognitive impairment and electroencephalographic alterations before and after liver transplantation: what is reversible? Liver Transpl 2014;20:977-86.

19. Colombari RC, de Ataide EC, Udo EY, et al. Neurological complications prevalence and long-term survival after liver transplantation. Transplant Proc 2013;45:1126-9.

20. Bernhardt M, Pflugrad H, Goldbecker A, et al. Central nervous system complications after liver transplantation: common but mostly transient phenomena. Liver Transpl
2015;21:224-32.

21. Kamath PS, Wiesner RH, Malinchoc M, et al. A model to predict survival in patients with end-stage liver disease. Hepatology 2001;33:464-70.

22. Stewart CA, Malinchoc M, Kim WR, et al. Hepatic encephalopathy as a predictor of survival in patients with end-stage liver disease. Liver Transpl 2007;13:1366-71.

23. Wong RJ, Gish RG, Ahmed A. Hepatic encephalopathy is associated with significantly increased mortality among patients awaiting liver transplantation. Liver Transpl 2014;20:1454-61.

24. Wong RJ, Aguilar M, Gish RG, et al. The impact of pretransplant hepatic encephalopathy on survival following liver transplantation. Liver Transpl 2015;21:873-80.

25. Garcia-Martinez R, Rovira A, Alonso J, et al. Hepatic encephalopathy is associated with posttransplant cognitive function and brain volume. Liver Transpl 2011;17:38-46.

26. Moore KA, Mc LJR, Burrows GD. Quality of life and cognitive function of liver transplant patients: a prospective study. Liver Transpl 2000;6:633-42.

27. Riether AM, Smith SL, Lewison BJ, et al. Quality-oflife changes and psychiatric and neurocognitive outcome after heart and liver transplantation. Transplantation. 1992;54:444-50.

28. Teperman LW. Impact of pretransplant hepatic encephalopathy on liver posttransplantation outcomes. Int J Hepatol 2013;2013:952828.
Cite this article as: Yoon JU, Yoo YM, Byeon GJ, Kim HJ, Choi EJ, Park S, Kim HY. The impact of pretransplant hepatic encephalopathy, model for end-stage liver disease (MELD) scale on long-term survival following deceased donor liver transplantation: a retrospective study. Ann Palliat Med 2021;10(5):5171-5180. doi: 10.21037/apm-21-21 